\title{
Multiscale Gradient Watersheds of Color Images
}

\author{
Iris Vanhamel, Ioannis Pratikakis, Member, IEEE, and Hichem Sahli, Associate Member, IEEE
}

\begin{abstract}
We present a new framework for the hierarchical segmentation of color images. The proposed scheme comprises a nonlinear scale-space with vector-valued gradient watersheds. Our aim is to produce a meaningful hierarchy among the objects in the image using three image components of distinct perceptual significance for a human observer, namely strong edges, smooth segments and detailed segments. The scale-space is based on a vectorvalued diffusion that uses the Additive Operator Splitting numerical scheme. Furthermore, we introduce the principle of the dynamics of contours in scale-space that combines scale and contrast information. The performance of the proposed segmentation scheme is presented via experimental results obtained with a wide range of images including natural and artificial scenes.
\end{abstract}

Index Terms-Anisotropic diffusion, color segmentation, dynamics of contours, scale-space, vector-valued gradient, watershed segmentation.

\section{INTRODUCTION}

I MAGE segmentation is an important task in computer vision that aims to partition the image into physically meaningful regions. Furthermore, it is an ill-defined problem, since what is really a physically meaningful region is often context dependent. It is difficult to construct a general region model that is based on image information such as grey-level, color, or texture. In spite of many considerable attempts, finding a method that can produce meaningful segments in a large variety of natural images remains a difficult task. In part this is due to the complexity of images. Image segments (regions) may differ in properties and these differences can be observed in some, but often not in all scales.

Segmentation algorithms for intensity images, may be divided into five broad categories: intensity thresholding [1], clustering [1], region growing [1], split and merge [1], and variational and Partial Differential Equation (PDE)-based approaches [2], [3], [4]. Approaches combining the last two categories have been also proposed by [5]-[8]. Even if many of these approaches have been extended to color images [9]-[14], the literature is not as rich as for grey-level images [1], [14], especially when we refer to segmentation algorithms integrating both superficial and deep image structure [5], [7], [15]-[20]. Segmentation algorithms that entail diffusion filtering, often employ the linear scale-space to address the multiscale framework [6], [7], [21], [22]. Nonlinear diffusion, on the other hand, is in general used as a preprocessing (enhancement) step for subsequent image segmentation [4], [15].

Manuscript received April 3, 2002; revised December 18, 2002. This work was supported by the Vlaams Instituut voor de bevordering van het Wetenschappelijk-Technologisch onderzoek in de industrie (IWT) under Grant 993222. The associate editor coordinating the review of this manuscript and approving it for publication was Dr. Philippe Salembier.

The authors are with ETRO/IRIS, Vrije Universiteit Brussel, B-1050 Brussels, Belgium (e-mail: iuvanham@etro.vub.ac.be).

Digital Object Identifier 10.1109/TIP.2003.811490
Hierarchical approaches are often used among segmentation methods. These approaches focus on the use of a hierarchical data structure representing the image at a number of scales. Each level of the hierarchy corresponds to a condensed version of the image at the previous level. This can be achieved by reducing the resolution of the image, leading to a regular hierarchical structure [23] or by merging segments in the image, leading to an irregular hierarchical structure [6], [7], [24]. Hierarchical segmentation can be achieved in two ways by using either top-down analysis or bottom-up analysis. Top-down analysis segments the image by successively refining the result from the previous coarse level, e.g., in [23]. In bottom-up methods, the segmentation is achieved by successively merging segments at each level until some conditions are satisfied [6], [24].

The analysis of multiscale watersheds [5], [8] resulted in a range of hierarchical segmentation methods varying from morphological pyramids [25]-[28] to multiscale hierarchies [6], [7], [22]. In the case of multiscale watersheds hierarchies, the duality between the watershed segments and regional minima of the gradient is exploited to either find markers for the watershed transformation or to establish a hierarchy between the watersheds using either the deep image structure individually [7] or the integration of both the superficial and deep image structure [6], [18].

In this paper, we strive toward an improved color segmentation scheme. Our method is based on the segmentation algorithm presented in [6], [29]. It uses the multiscale structure built by vector-valued nonlinear diffusion filtering [30] to measure and incorporate various perceptual properties such as the dynamics of contours [31], smooth segments and detailed segments. This results in a hierarchy among segments produced by the color gradient watershed, and detects meaningful segments in a variety of natural and artificial images. The proposed hierarchical segmentation scheme is addressed within the framework of nonlinear diffusion filtering. Unlike linear diffusion filtering, anisotropic diffusion filtering is guided by additional information that leads to intraregion smoothing and edge enhancement [32]-[34]. To deal with vector-valued data, such as color images, we propose a vector-valued diffusion scheme [30], as an extension to the regularized version of the anisotropic diffusion proposed by Perona and Malik [32], [33]. Moreover, the numerical model that was given in [32] is replaced by the more efficient Additive Operator Splitting (AOS) method [35]. Our segmentation scheme consists of two modules, the Salient Measure Module and the Hierarchical Levels Retrieval Module. The Salient Measure Module uses the dynamics of multiscale gradient watersheds, which integrates both the superficial and deep image structure, to valuate the contours. The Hierarchical Levels Retrieval Module uses a bottom-up hierarchical analysis. The merging at each level of the hierarchy is performed based on a color homogeneity criterion. The proposed integration embodies three perceptual features, 
namely contrast, homogeneity and scale. We demonstrate the quality of our segmentations taking into account the following aspects: i) the scale-space generator and the linking scheme; ii) the evolution of the dynamics of contours in scale-space; iii) the hierarchy among the gradient watersheds obtained in the case of using the superficial image structure, the deep image structure, as well as the integration of both (the dynamics of contours in scale-space); and iv) the hierarchical levels retrieval and the optimal hierarchical level selection.

This paper is organized as follows. Section II is dedicated to a detailed description of the segmentation scheme. In Section II-A, the gradient watershed transformation for color images is summarized. Section II-B deals with the scale-space generation. The Salient Measure Module is described in Section II-C. This module embodies the scheme for linking information across the scale-space stack (Section II-C2) and explains the multiscale feature used for contour valuation (Sections II-C1 and II-C3). The Hierarchical Levels Retrieval Module is described in Section II-D. A comparative study of the Gaussianbased and the anisotropic diffusion-based multiscale segmentation scheme is given and illustrated with experimental results in Section III. Finally, conclusions concerning the performance of the proposed segmentation method and the continuation of our research are given in Section IV.

\section{Hierarchical Multiscale Segmentation}

Our main goal is to create a hierarchy among the color gradient watersheds that preserves the topology of the watershed lines at the localization scale (the finest scale in the scale-space stack) and extracts homogeneous objects of a larger scale. Let us first define what we mean by a hierarchy. Let $P^{0}=\left\{S_{1}, S_{2}, \ldots, S_{n}\right\}$ be the initial partitioning of the image at the localization scale after the application of the color gradient watershed transformation. A hierarchical level $k\left(H L_{k}\right)$ is defined as the partitioning $P^{k}=\left\{S_{1}^{k}, S_{2}^{k}, \ldots, S_{m_{k}}^{k}\right\}$ which preserves the inclusion relationship $P^{k} \supseteq P^{k-1}$, implying that each segment of the set $P^{k}$ is a disjoint union of segments from the set $P^{k-1}$. A hierarchy of partitions is defined as a family which consists of all the hierarchical levels $H L_{k}$, where $k \in[0, K]$. It corresponds to a hierarchy of Region Adjacency Graphs, $\mathcal{G}_{k}\left(P^{k}, A^{k}\right)$, that are generated by applying successive mergings.

Different approaches exist in the literature for constructing a hierarchy of partitions defined on the basis of the watershed transformation that retrieve information from the superficial image structure. Hierarchical approaches emanating from a Mathematical Morphology (MM) framework consist of i) the waterfall algorithm [36], ii) the dynamics of minima [37], iii) extinction value [38] and iv) the dynamics of contours [31]. This enumeration corresponds to the chronological order of their appearance in the literature. Essentially, this hierarchy can be interpreted as a set of region adjacencies, wherein an ordering is determined by a valuation, which can be controlled by the relative altitudes of the minima (dynamics of minima, and dynamics of contours) or by the relative heights of the watershed lines (waterfall). The extinction value introduces area to the concept of dynamics. Comparisons of these approaches can be found in [24], [31], and [39].

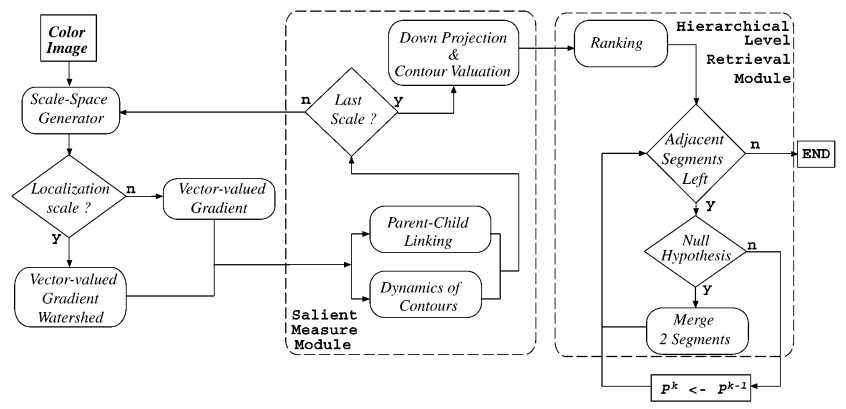

Fig. 1. Schematic diagram for the proposed hierarchical segmentation scheme for color images.

The proposed hierarchical segmentation scheme, depicted in Fig. 1, consists of two basic modules. The first module (Salient Measure Module-Section II-C) is dedicated to attribute a saliency measure to each contour arc at the localization scale taking into account the whole scale-space stack. The entire process to retrieve the saliency measure for the color gradient watersheds requires three steps: i) scale-space stack generation by vector-valued nonlinear diffusion filtering (Section II-B). ii) Linking (Section II-C2). At each scale the color gradient of the image is estimated. At the localization scale, the watershed transformation is performed to identify the position of all the contours in the image. At the higher scales, the duality between the regional minima of the color gradient and the catchment basins of the watershed is exploited to make a robust region based parent-child linking scheme. iii) Contour valuation by downward projection (Section II-C3). The dynamics of contours in scale-space are used to valuate the contours detected at the localization scale.

The second module (Hierarchical Levels Retrieval ModuleSection II-D) identifies the different hierarchical levels through a hypothesis testing criterion. Starting from the watershed segmentation at the localization scale, a successive merging operation is performed until a stopping criterion is satisfied. The merging of adjacent regions is based upon a color similarity measure. While the merging sequence (order) is given by the valuation (dynamics of contours in scale-space) of the contours.

\section{A. Color Gradient Watersheds}

The watershed transformation is a morphological tool dedicated to image segmentation. It can be considered as a topographic region growing method. In the watershed transformation, pixel values are considered as topographic data characteristics of a relief, thus, the value of each pixel denotes the elevation of the point [36], [40]. The basic idea of the watershed construction is to create an influence zone for each regional minima of the image [41]. In that respect, a one-to-one mapping is attributed between the regional minima and the catchment basin.

In the case of segmentation, the watershed transformation is often applied on the gradient magnitude of an image in order to guide the watershed lines to follow the crest lines and the real boundaries of the objects. Therefore, from now on, we will refer to gradient watersheds, thus explicitly implying that we have retrieved the watershed lines from the modulus of the gradient image. In grey level images, the modulus of the gradient is a scalar function of the coordinates and expresses the distance 
between neighboring pixels with respect to their intensity. In the case of color images, the modulus of the gradient expresses the color distance in the chosen color space using a certain metric.

Approaches that estimate the color gradient can be classified into three main categories: i) fusion methods; ii) statistics-based methods; and iii) gradient tensor or gradient vector methods. The first class of methods estimate the gradient of each color component separately and fuse the results [10], [42]. The second category of methods use statistical features to estimate color differences [43]. The above mentioned approaches do not consider color images as vector-valued functions for the gradient estimation. In the third set of methods an explicit formulation of the vector-tensor is given [44], [45]. This allows interaction among the color channels and results in a more accurate description of the first order derivatives. In this work, the color gradient, in the $Y U V$ color space, is estimated using the Di Zenzo vector-valued gradient [44] in conjunction with the Sobel operators for the estimation of the partial derivatives of each color component.

\section{B. Scale-Space Generation}

The purpose of segmentation is the partitioning of an image into meaningful objects. Since real-world objects are meaningful only in a certain range of scale [46], it is therefore natural to include the multiscale nature of images in the segmentation process. In controlled circumstances the scale of the objects in the scene is known, contrary to most natural scene images [46], [47]. For these images it makes sense to analyze the image at all scales simultaneously; the analysis of the deep image structure. The multiscale nature of images can be represented in various ways. Scale-space theory has a firm mathematical foundation and all known scale-space models can be derived from the diffusion equation

$$
\delta_{t} \mathbf{u}=\operatorname{div}(\mathbf{D} \nabla \mathbf{u})
$$

where $t$ is the scale parameter, $\mathbf{u}$ is the scale-space image of an image $\mathbf{f}$, and $\mathbf{D}$ is the diffusion tensor.

1) Gaussian Scale-Space: In linear scale-space methods, $\mathbf{D}$ is a scalar value and is referred to as diffusivity. The linear scale-space is commonly used in segmentation [6], [7], [21]-[23], [48]. The scale-space image is obtained by convolving the original image with Gaussian kernels of increasing width. The kernel size $(\sigma)$ and the scale-parameter $(t)$ are related by $\sigma=\sqrt{2 t}$. Linear scale-spaces exhibit useful properties such as the semi-group property, which results in fast methods and scale-invariance [34]. The Gaussian diffusion for vector-valued images is performed in each channel separately. Fig. 2 shows an example of the Gaussian scale-space stack applied to a color image.

2) Anisotropic Diffusion: The inherent problems of the linear scale-space methods led to the investigation of their nonlinear counterparts. In the nonlinear methods, extra information is added to guide the diffusion process. Perona and Malik introduced an anisotropic diffusion filtering for scalar images that avoids blurring and ensures well-localized edges [32]. In later works, it was spatially regularized to guarantee well-posedness [33], [34]. In the literature, many works report on the use of anisotropic diffusion for scalar images, however there is not much reported on diffusion for

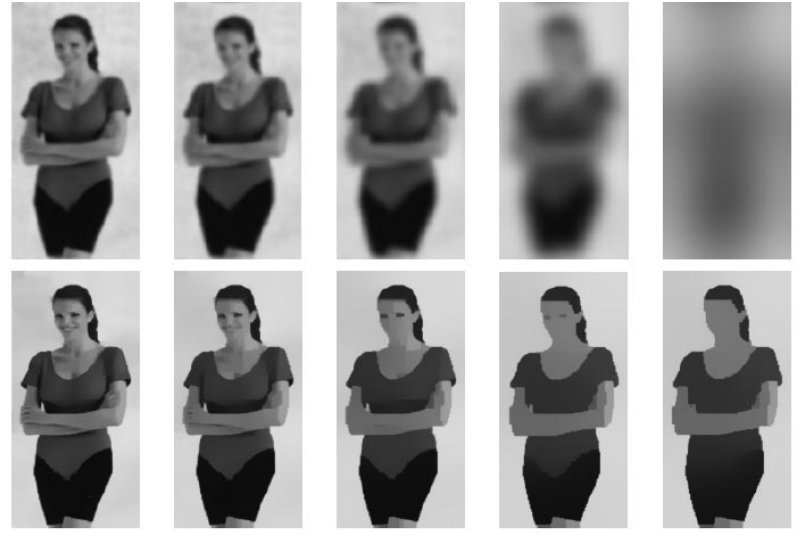

Fig. 2. Scale-space stack in $Y U V$ color-space. Top: Gaussian scale-space stack from left to right with $s=0,3,9,18,31, \tau_{o}=0.3$, and $\delta \tau=0.1$. Bottom: CLMC stack from left to right with $s=0,5,10,15,17, \tau_{o}=2.10$, and $\delta \tau=0.1$.

vector-valued images. Whitaker et al. introduced the general framework for vector-valued diffusion in [30]. The latter is a straightforward extension of the work presented in [32] to a system of coupled diffusion equations. Each channel is diffused separately using a diffusion tensor which is estimated using information of all channels. Chambolle et al. [49] present some preliminary results concerning color images by comparing the use of coupled diffusion equations with a not coupled system. An application of the latter can be found in [14], where the luminance and chromatic information are diffused separately. Sapiro et al. estimate an anisotropic flow based upon the gradient in vector-valued images [44]. In their approach the diffusion is perpendicular to the direction of the maximal rate of change [50], [51].

In this paper, we use an anisotropic diffusion for color images, which was initially described in [32] for gray level images and which was modified by Catté, Lions, Morel, and Coll [33] to ensure well-posedness. This filter will be referred to as CLMC throughout the rest of paper. For our scheme, we use a system of coupled diffusion equations where the diffusion tensor is estimated using the Euclidean distance between the color vectors of neighboring pixels. We use the Additive Operator Splitting numerical scheme (AOS) [35]. It has an increased efficiency compared to the explicit scheme [32] and is easier to solve than the semi-implicit scheme in higher dimensions [33].

Let $\Omega:=\left(0, \mathbf{b}_{1}\right) \mathrm{x} \cdots \mathrm{x}\left(0, \mathbf{b}_{M}\right)$ be a $M$-dimensional image domain and consider a vector-valued image $f$ as a bounded mapping from $\Omega$ into the real vector $\mathbb{R}^{m}$; with $M=2$ and $m=3$ in the case of a color image. Then the CLMC filter obtains a filtered image $\mathbf{u}(\mathbf{x}, t)$ of $\mathbf{f}(\mathbf{x})$ as the solution of (1), for which the original image $\mathbf{f}$ is the initial state $\mathbf{u}(\mathbf{x}, 0)$, and for which reflecting boundary conditions are assumed on the image boundary $\left(\delta_{n} \mathbf{u}=0\right.$ on $\left.\delta \Omega\right)$. The diffusion tensor $\mathbf{D}$ is a function that favors wide regions over smaller ones and is described as follows:

$$
\mathbf{D}=\mathbf{D}\left(\left|\nabla\left(\mathbf{u}_{\sigma_{r}}\right)\right|^{2}\right)=\frac{1}{1+\frac{\left|\nabla\left(\mathbf{u}_{\sigma_{r}}\right)\right|^{2}}{\lambda^{2}}}
$$

where $\sigma_{r}$ is the size of the Gaussian kernel used for the spatial regularization and was set to 0.5 during all our experiments. 
The constant $\lambda>0$ is a contrast parameter that separates forward (low contrast) from backward (high contrast) diffusion. The value of $\lambda$ is problem dependent and is determined experimentally for most applications. In this work, an automatic estimation of the contrast parameter is proposed: We assume that pixels, for which $\left|\nabla\left(\mathbf{u}_{\sigma_{r}}\right)\right|^{2} \geq 10 \%$ of the maximum of the corresponding histogram, are edge pixels. Subsequently the amount of diffusion at those pixels should be as small as possible, e.g., $\varepsilon=0.01$. The contrast parameter is obtained as follows:

$$
\lambda=\sqrt{\frac{\varepsilon}{1-\varepsilon} \cdot L}
$$

where $L$ denotes $10 \%$ of the maximum of the histogram of $\left|\nabla\left(\mathbf{u}_{\sigma_{r}}\right)\right|^{2}$.

The majority of the numerical schemes are explicit and use finite differences. For a vector-valued image and considering a vector-matrix notation, the explicit numerical scheme is the following:

$$
u_{i}^{t+1}=\left(I+\tau \cdot A\left(\left|\nabla\left(\mathbf{u}_{\sigma_{r}}^{t}\right)\right|^{2}\right) u_{i}^{t}\right.
$$

where $u_{i}$ is a color channel, $I \in \mathbb{R}^{M}$ is the unit matrix and $\tau$ is the time step. The matrix $A\left(\left|\nabla\left(\mathbf{u}_{\sigma_{r}}^{t}\right)\right|^{2}\right)=\left[a_{\mathbf{x x}_{j}}\right]$, which contains the diffusion values, is defined as follows:

$$
a_{\mathbf{x x}_{j}}= \begin{cases}D_{j}\left(\left|\nabla \mathbf{u}_{\sigma_{r}}^{t}\right|^{2}\right) & \left(\mathbf{x}_{j} \in \mathcal{N}(\mathbf{x})\right) \\ -\sum_{\mathbf{x}_{n} \in \mathcal{N}(\mathbf{x})} D_{n}\left(\left|\nabla \mathbf{u}_{\sigma_{r}}^{t}\right|^{2}\right) & \left(\mathbf{x}_{j}=\mathbf{x}\right) \\ 0 & \text { (otherwise) }\end{cases}
$$

where $\mathbf{x}$ and $\mathbf{x}_{j}$ are pixels, $\mathcal{N}(\mathbf{x})$ is the neighborhood of pixel $\mathbf{x}$ and contains the pixels $\mathbf{x}_{j}$ with $j=N$ (orth), $S$ (outh), $E$ (ast) and $W$ (est) on a square lattice. $D_{j}\left(\left|\nabla \mathbf{u}_{\sigma_{r}}^{t}\right|^{2}\right)$ is the diffusivity at pixel $\mathbf{x}$ estimated for orientation $j$, and $\Delta_{j}\left[u_{i}\right]_{\mathbf{x}}^{t}=u_{i}^{t}\left(\mathbf{x}_{j}\right)-u_{i}^{t}(\mathbf{x})$ denotes the nearest neighbor difference. The values $D_{j}\left(\left|\nabla \mathbf{u}_{\sigma_{r}}^{t}\right|^{2}\right)$ are calculated by applying (2) to the Euclidean difference between the color vectors of the corresponding pixels $\mathbf{x}$ and $\mathbf{x}_{j}$. To ensure stability for the discrete scheme [(4)], the time step $(\tau)$ has to be in $] 0,1 / 4[$. Hence, the whole filtering process becomes rather time consuming. In the case of the semi-implicit numerical method, the time step is not restricted but the scheme is difficult to solve in higher dimensions. Therefore, Weickert et al. [34], [35] proposed to split the semi-implicit method into $\xi$ one-dimensional problems and to sum the results

$$
u_{i}^{t+1}=\frac{1}{\xi} \sum_{l=1}^{\xi} \frac{u_{i}^{t}}{\left(I-\xi \cdot \tau \cdot A_{l}\left(\left|\nabla \mathbf{u}_{\sigma_{r}}^{t}\right|^{2}\right)\right)}
$$

where $\xi$ denotes the number of directions related to the type of the grid connectivity used. In this work, a 4 connectivity was chosen, hence $\xi=2 . A_{l}\left(\left|\nabla \mathbf{u}_{\sigma_{r}}^{t}\right|^{2}\right)$ consist of the diffusion coefficients, as defined in (5), using a neighborhood $\mathcal{N}_{l}(\mathbf{x})$ in the corresponding direction $l$. In our experiments, the time step $(\tau)$ never exceeds 5 . This way, we avoid visible errors in the approximation of the diffused image [35]. An example of the scale-space stack, obtained using the presented CLMC diffusion, is shown in Fig. 2.
3) Scale-Space Sampling: The sampling of the scale-space stack provides a natural sampling and ensures scale invariance for the linear scale-space. It follows a linear and dimensionless scale parameter $\delta \tau$, which is related to the size of the Gaussian kernel $\sigma$ by [34]

$$
\sigma_{s}=e^{\tau_{o}+s \delta \tau}
$$

where $s$ denotes the scale quantization level and $t_{s}=\sigma_{s}^{2} / 2$. For the nonlinear scale-space there is no global scale parameter because the amount of diffusion changes locally. Nevertheless, since $\left|D_{j}\left(\left|\nabla \mathbf{u}_{\sigma_{r}}^{t}\right|^{2}\right)\right| \leq 1$, we can apply the same scheme. In this case, the time step $\tau$ in (4) and (6) is retrieved as follows:

$$
\begin{aligned}
\tau & =t_{s_{i+1}}-t_{s_{i}} \\
& =\frac{e^{2\left(\tau_{o}+i \delta \tau\right)}}{2} \cdot\left(e^{2 \delta \tau}-1\right) .
\end{aligned}
$$

The localization scale $\left(s_{o}\right)$, which is derived from $\tau_{o}[(7)$ and (8)], should contain all information in the image with a minimal amount of noise. Furthermore, the application of the color gradient watershed should result in accurate detection of the watersheds. In this work, the localization scale is determined empirically.

\section{Salient Measure Module}

The aim of this module is to valuate the arcs of the Region Adjacency Graph $(R A G), \mathcal{G}_{0}\left(P^{0}, A^{0}\right)$, at the localization scale $\left(s_{0}\right) . \mathcal{G}_{0}$ is obtained after the application of the color gradient watershed transformation, with $P^{0}=\left\{S_{1}, S_{2}, \ldots, S_{n}\right\}$ the segments (regions), and $a \in A^{0}$ the arcs separating pairs of adjacent segments $\left(S_{i}, S_{j}\right)$.

The proposed valuation relies on the concept of the dynamics of contours in scale-space [6], which incorporates a region linking that has been advocated by a study of the topological changes of the critical point configuration in linear scale-space [7], [29]. The main motivation for the cooperation of the watershed analysis and the scale-space is the duality of the catchment basins of the watershed with their respective minima in the gradient image and the simplification process, which occurs during the evolution of the scale-space stack.

1) Dynamics of Contours: The principle of dynamics of contours [31] uses the principle of dynamics of minima [37] as an initial information for the common contour valuation of adjacent regions. The additional information that is used, is based upon the tracking of the flooding history. In such a way, a contour valuation can be found by comparing the dynamics of minima between the segments, which have reached the contour of interest during a flooding. The dynamics of a minimum $m_{1}$ is easily defined with a flooding scenario. Let $h$ be the altitude of the flood when for the first time a catchment basin with a deeper minimum $m_{2},\left(m_{2}<m_{1}\right)$ is reached. The dynamics of $m_{1}$ is then equal to $h$-altitude $\left(m_{1}\right)$. Each catchment basin is attributed the value of the dynamics of its minimum. The contour valuation which is attributed to each common border of the segments is denoted as [31]

$$
D C\left(S_{i}, S_{j}\right)=\min _{q} \max _{a_{q} \in B_{q}}\left\{f(a)-f\left(a_{q}\right)\right\}
$$


where $a$ denotes the lower point (saddle point) of the common border between segments $S_{i}$ and $S_{j}, f$ is the function on which the flooding is applied, and $B_{q}$ denotes an open connected component that belongs to the topological open set $B a s(a)$ which is defined as

$$
\begin{aligned}
\operatorname{Bas}(a):=\{b \mid \exists \gamma, \gamma(0)= & a, \gamma(1)=b, \\
& f(\gamma(l))<f(a) \forall l \in] 0,1]\} .
\end{aligned}
$$

Linking Scheme: The linking scheme aims to track the regional minima in the gradient through the scale-space. The linking process is applied using the approach proposed in [29]. Here, the linking of the minima for successive scales is applied by using the proximity criterion [46]. This criterion is limited for projected minima of scale quantization level $s_{i}$ inside the same geodesic influence zone $i z_{A}^{s_{i+1}}\left(B_{q}\right)$ of a connected component $B_{q}$ of $B$ in $A$ at scale quantization level $s_{i+1}$

$$
\begin{aligned}
i z_{A}^{s_{i+1}}\left(B_{q}\right)=\{p \in A, \forall j \in[1, k] /\{q\}, \\
\\
\left.d_{A}\left(p, B_{q}\right)<d_{A}\left(p, B_{j}\right)\right\} .
\end{aligned}
$$

Any regional minimum of the set $\left\{m^{s_{i}}\right\}$, that is spatially projected on the geodesic influence zone $i z_{A}^{s_{i+1}}\left(B_{q}\right)$ at scale quantization level $s_{i+1}$, will be linked with the regional minimum $m_{q}^{s_{i+1}}$. The projected minimum of the set $\left\{m^{s_{i}}\right\} \in i z_{A}^{s_{i+1}}\left(B_{q}\right)$, which is the closest to the minimum $m_{q}^{s_{i}+1}$, is considered as the father. The rest of the projected minima onto the same influence zone are considered annihilated. Closeness is defined with respect to the topographic distance which is a natural distance measure following the steepest gradient path inside the catchment basin. We have to mention that we use inverse ordered queues to guide all regional minima of the set $\left\{m^{s_{i}}\right\}$ toward the set $\left\{m^{s_{i+1}}\right\}$. This way, we avoid problems caused by the presence of plateaus.

Fig. 3 illustrates the linking of the regional minima between successive scales. In Fig. 3(a), the regional minima at scale quantization level $s_{i}$ that are spatially projected on the level $s_{i+1}$, are shown. Furthermore, the watershed lines that delimit the influence zones at scale quantization level $s_{i+1}$ are depicted. Fig. 3(b) shows the regional minima at scale quantization level $s_{i+1}$. The linking between the regional minima $\left\{m^{s_{i}}\right\}$, at scale quantization level $s_{i}$, and the minima $\left\{m^{s_{i+1}}\right\}$, at scale quantization level $s_{i+1}$, is given in Fig. 3(c). Note that, at each scale quantization level, after the linking stage, we attribute a label to the minima with respect to their linking ability: i) Either the minimum is annihilated/merged and will not be considered in the linking of the next levels or ii) it does not interact with other singularities and takes up the role of the father label for all the minima situated at the same influence zone.

At the end of the linking step, for each couple of neighboring segments $\left(S_{i}, S_{j}\right)$ that share a common border at the localization scale, a linkage list $\Lambda\left(S_{i}, S_{j}\right)$ is constructed. Fig. 4(a) shows such a list for $S_{i}=A$ and $S_{j}=B$. This list provides the following information: Segment $F$ is attributed to a Root for segments $A$ and $B$ at $s_{4}$. This has occurred because i) at $s_{2}$, segment $A$ has been linked to segment $D$, which has been linked to segment $F$ at $s_{4}$ and, ii) at $s_{1}$, segment $B$ has been linked to segment $C$, which at $s_{3}$, has been linked to segment $E$. The latter has been linked to segment $F$ at $s_{4}$.

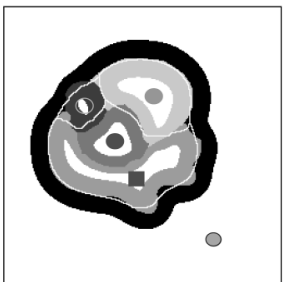

(a)

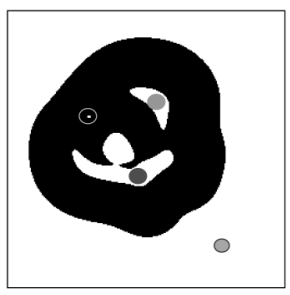

(b)

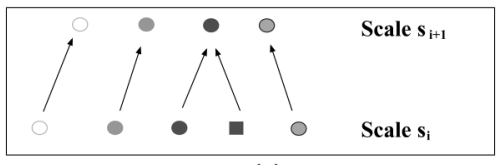

(c)

Fig. 3. Linking of the regional minima between successive scales.

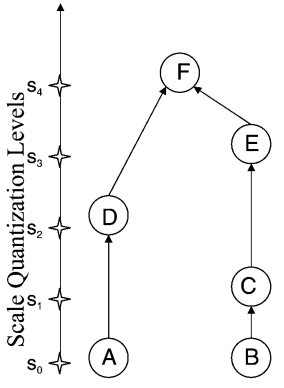

(a)

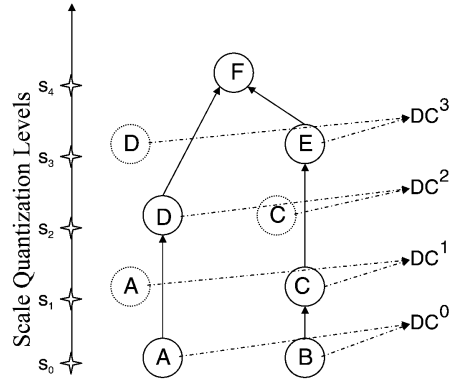

(b)
Fig. 4. Down projection and Contour valuation [the linkage list $\Lambda\left(S_{i}, S_{j}\right)$ ].

2) Contour Valuation by Down-Projection, Dynamics of Contours in Scale-Space: The next step is to valuate the gradient watersheds at the localization scale $s_{0}$. For each segment couple $\left(S_{i}^{\Lambda\left(S_{i}, S_{j}\right)_{m}}, S_{j}^{\Lambda\left(S_{i}, S_{j}\right)_{m}}\right)$, appearing at the branch $m$ (scale quantization level $s_{m}$ ) of the linkage list $\Lambda\left(S_{i}, S_{j}\right)$, we compute the dynamics of contours $D C_{\Lambda\left(S_{i}, S_{j}\right)_{m}}^{m}$ [see Fig. 4(b)]. $D C_{\Lambda\left(S_{i}, S_{j}\right)_{m}}^{m}$ expresses how much contrasted the adjacent regions $\left(S_{i}, S_{j}\right)$ are at the scale quantization level $s_{m}$. Finally, the dynamics of contours in scale-space $(D C S)$ for the adjacent region couple $\left(S_{i}, S_{j}\right)$ is defined as the sum for all valuations $D C_{\Lambda\left(S_{i}, S_{j}\right)_{m}}^{m}$ during the evolution in scale-space

$$
D C S\left(S_{i}, S_{j}\right)=\sum_{m=0}^{N-1} D C_{\Lambda\left(S_{i}, S_{j}\right)_{m}}
$$

where $N$ denotes the branch of the linkage list $\Lambda\left(S_{i}, S_{j}\right)$ where the contour formed by the region couple $\left(S_{i}, S_{j}\right)$ is annihilated. The difference $\Delta s=s_{N}-s_{0}$ is the corresponding scale-space lifetime (SLT).

As output of the Salient Measure Module, the $\operatorname{arcs} a=$ $\left(S_{i}, S_{j}\right) \in A^{0}$, of the RAG $\mathcal{G}_{0}\left(P^{0}, A^{0}\right)$, are ranked according to their saliency $\operatorname{DCS}\left(S_{i}, S_{j}\right)$.

\section{Hierarchical Levels Retrieval Module}

Having as inputs the initial RAG, $\mathcal{G}_{0}$, and the ranked saliency values $\left\{D C S\left(S_{i}, S_{j}\right)\right\}$, this module provides a hierarchy $\mathcal{G}_{0}, \mathcal{G}_{1}, \ldots, \mathcal{G}_{k}, \ldots, \mathcal{G}_{K}$ of Region Adjacency Graphs. Each hierarchical level $\mathcal{G}_{k}\left(P^{k}, A^{k}\right)$, for $k>0$, is retrieved by successively merging adjacent regions of $\mathcal{G}_{k-1}$, until the violation 
of a stopping criterion. This criterion uses the Hotteling's $T^{2}$ hypothesis test [17], while the merging, of adjacent regions, is based on the Mahalanobis distance between their color distributions (homogeneity constraint).

For our problem, the hypothesis test is defined as

$H_{0}^{k}$ : Two adjacent regions at level $k-1$ belong to the same region at level $k$;

$H_{1}^{k}$ : Two adjacent regions at level $k-1$ belong to different regions at level $k$;

where $H_{0}^{k}$ is the null hypothesis and $H_{1}^{k}$ denotes the alternative hypothesis. The consecutive hierarchical levels are constructed based on the ability of each merged couple to satisfy the null hypothesis, during the hypothesis testing, as we sequentially scan the ranked saliency values.

Let $S_{i}^{k-1}$ and $S_{j}^{k-1}$ two adjacent regions at level $k-1$, with cardinalities $n_{i}^{k-1}$ and $n_{j}^{k-1}$, and mean color vectors $\mu_{i}^{k-1}$ and $\mu_{j}^{k-1}$, respectively. Additionally we denote by $\left(S_{i}^{k-1} \cup S_{j}^{k-1}\right)^{k}$ the merged region at level $k$, with cardinality $n_{i j}^{k}$ and color covariance matrix $\Sigma_{i j}$. We formulate our hypothesis testing as a Hotteling's $T^{2}$ test, which is the generalization of the $\chi^{2}$ test approach for multivariate analysis

$$
\begin{aligned}
& H_{0}^{k}: \tilde{F}_{(i j)^{k}}^{2}<F_{\left(3, n_{i j}^{k}-4\right)}(\alpha) \Rightarrow P(i, j)=\text { true } \\
& H_{1}^{k}: \tilde{F}_{(i j)^{k}}^{2} \geq F_{\left(3, n_{i j}^{k}-4\right)}(\alpha) \Rightarrow P(i, j)=\text { false. }
\end{aligned}
$$

$P(i, j)$ is a decision function which is true if the updated regions $i$ and $j$ belongs to the same region at level $k$, while otherwise it is false. The statistic

$\tilde{F}_{(i j)^{k}}^{2}=\frac{n_{i j}^{k}-4}{3\left(n_{i j}^{k}-2\right)} \frac{\left(\mu_{i}^{k-1}-\mu_{j}^{k-1}\right)^{t} \Sigma_{i j}^{-1}\left(\mu_{i}^{k-1}-\mu_{j}^{k-1}\right)}{\frac{1}{n_{i}^{k-1}}+\frac{1}{n_{j}^{k-1}}}$

is based on the Mahalanobis distance and used to decide whether or not to merge the adjacent regions. $F_{(m, l)}(\alpha)$ is the critical value of the Fisher distribution, with $(m, l)$ degrees of freedom, at significance level $\alpha$. The value of $\alpha$ is the probability of rejecting the null hypothesis.

Upon this definition it is critical to say that for every level transition, from $k-1$ to $k$, we update the statistic [(14)] used to formulate the hypothesis in (13). During our experiments $\alpha$ is set to 0.05 , and the mean vectors and the covariance matrix for the merged regions are computed using the statistics of the constituent partitions (from the localization scale). As a last note, it has to be mentioned that, to ensure meaningful statistics before applying the hierarchical levels retrieval, small regions at the localization scale are merged with their most coherent neighbor. A most coherent neighbor is defined as the one that shares the boundary (arc) with the lowest $D C S$ value. Fig. 9 shows the obtained hierarchical levels for the image in Fig. 2.

\section{EXPERIMENTAL RESULTS}

In this Section, we analyze the proposed segmentation scheme focusing on the following: i) the scale-space generator and the linking scheme; ii) the evolution of the dynamics of contours in scale-space; iii) the hierarchy among the gradient watersheds obtained in the case of using the principle of dynamics of contours at the localization scale $\left(D C^{s_{0}}\right)$, the

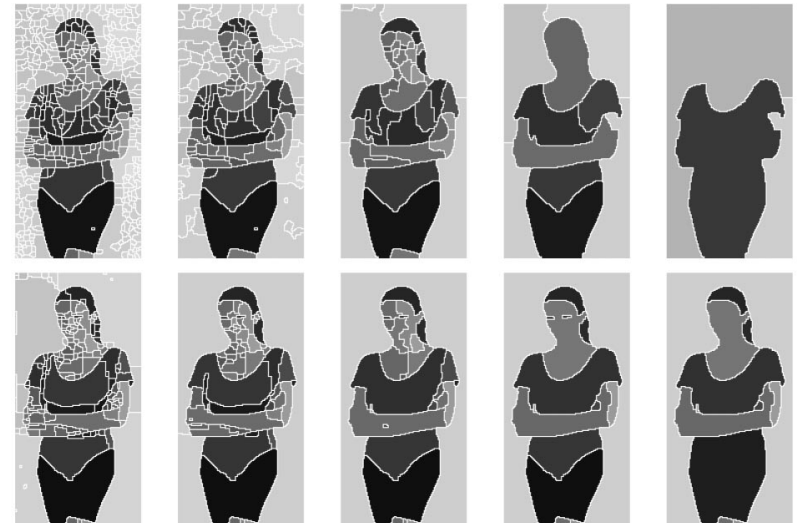

Fig. 5. Segmented scale-space stack after the projection of the regional minima. Top: Gaussian diffusion, from left to right: $s=0,3,9,18,31$ with $456,157,43,14$, and 2 segments. Bottom: CLMC diffusion. $s=0,5,10,15$, 17 with $176,71,31,16$, and 13 segments.

dynamics of contours in scale-space (DCS), as well as using the scale-space lifetime (SLT) alone; and iv) the hierarchical levels retrieval and the optimal hierarchical level selection.

i) Two scale-space generators have been analyzed, namely the Gaussian scale-space and the regularized Perona and Malik anisotropic diffusion (CLMC). In Fig. 2 the scale-space stack produced by both generators, is shown. Through all our experiments the localization scale is determined empirically. In the case of Gaussian diffusion, we have chosen $\tau_{0}=0.3$ that ensured a good localization of the color gradient watersheds. Unlike the Gaussian diffusion, the CLMC diffusion does not suffer from the edge dislocation problem. Therefore, the localization scale has been set at a larger scale $\left(\tau_{0}=2.10\right)$ that has an optimal noise reduction whilst retaining all image features. The segmentation results at the localization scale are shown in the first column of Fig. 5. It is clearly shown that, the CLMC diffusion exhibits less oversegmentation.

To treat the scale-space as a single entity we apply a linking scheme (Section II-C2) that enables the correspondence of segmented regions at successive scales. Furthermore, linking avoids the problem of dislocation of borders that is inherent to the Gaussian scale-space. To provide the information reduction regarding the number of regions that occurs in scale-space, the produced segmentations after the projection of the linked region entities at the localization scale, are shown in Fig. 5. Furthermore, Fig. 6 shows the amount of linked regional minima as a function of the scale-space stack. As it can be seen, the Gaussian diffusion reduces the amount of information faster. Nevertheless, for the CLMC diffusion, significant features remain longer in scale-space. This can be seen in Fig. 5 that shows the longer in time preservation of the "girl" eyes.

ii) We present a study for three different representative types of contours (Fig. 7) about their evolution in scale-space taking into account the following: dynamics of contours $\left(D C^{i}\right)$, the grey value of the regional minima and the corresponding saddle point for the color gradient. Our aim is not only to show the behavior of the dynamics of contours during evolution but also to demonstrate the behavior of the corresponding regional minima and saddle points that contribute to this valuation. The first contour is a strong one, situated between the background and the 


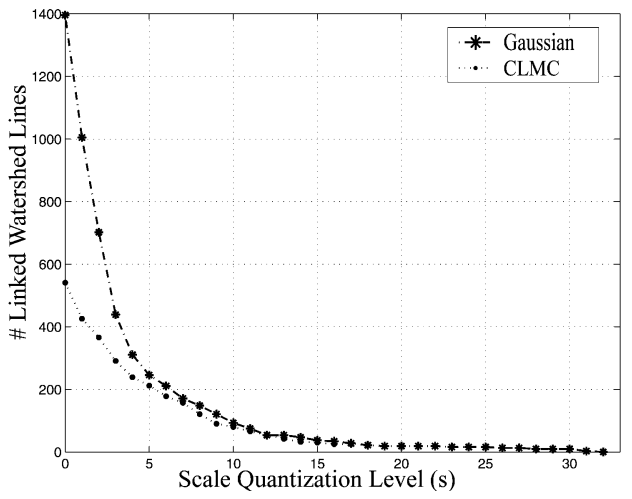

Fig. 6. Information reduction: Number of linked regional minima in scale-space.
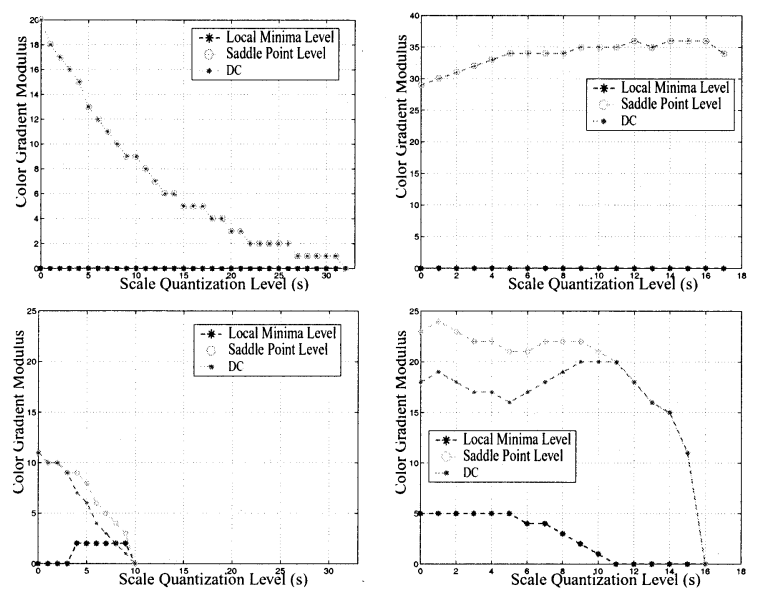

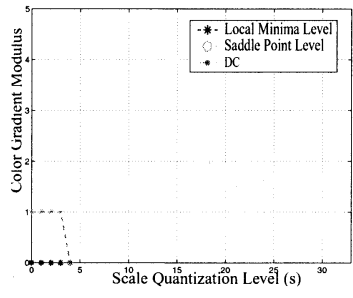

(a)

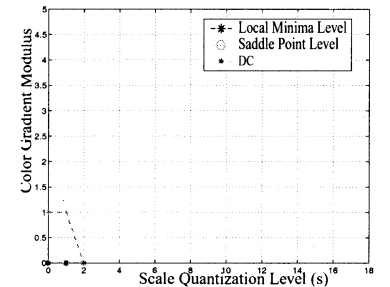

(b)
Fig. 7. Evolution of the Dynamics of Contours in scale-space: (a) Gaussian diffusion and (b) $C L M C$ diffusion in the case of (first row) a strong contour, (second row) a small but salient contour, and (third row) a weak contour.

right leg of the girl. The second contour is small but salient, separating the left eye from the face. The third contour is very weak separating two segments in the background.

As it can be seen in Fig. 7(c), the weak contours disappear quickly for both diffusions. In the case of salient contours [Fig. 7(a), (b)], we can observe their preservation for longer periods along with their enhancement (increase of dynamics of contours) for the CLMC diffusion. Furthermore, CLMC diffusion attempts to smooth out the region's interior before removing the contour. This explains the decrease of the regional minima's level and the corresponding increase in the dynamics of contours. In contrary a fast decrease occurs for the Gaussian diffusion that is related to the fact that the level of the corresponding saddle point is decreasing while the level of the regional minima increases.

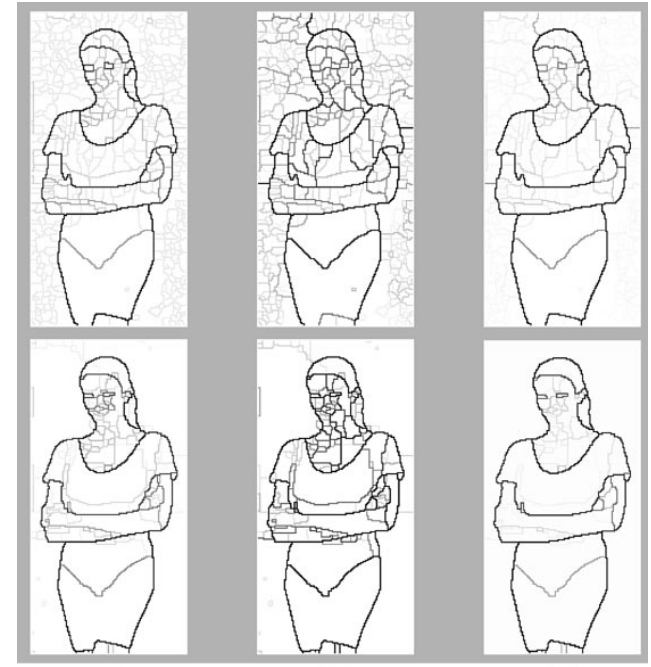

(a)

(b)

(c)

Fig. 8. Saliency maps for the Gaussian diffusion (top) and the CLMC diffusion (bottom) using (a) $D C^{0}$, (b) $S L T$, and (c) $D C S$.
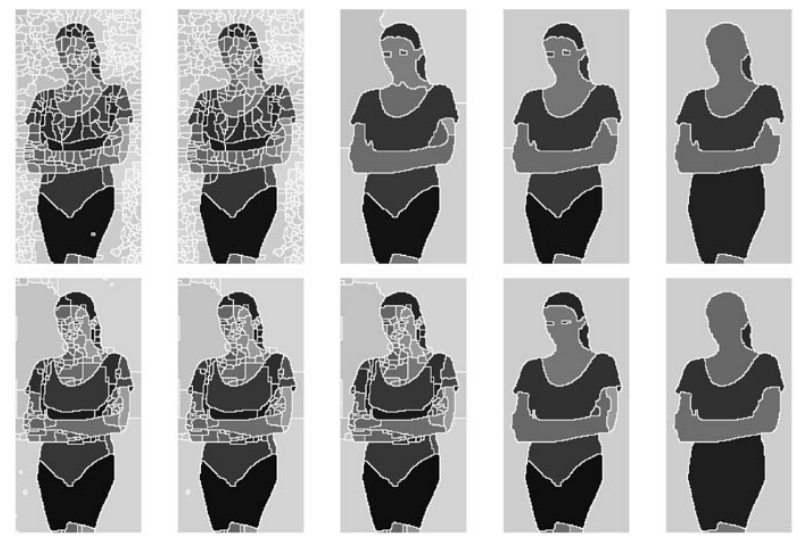

Fig. 9. Hierarchical Levels $P^{k}$ in $Y U V$ color-space. Top: Gaussian diffusion, from left to right: $k=0,1,8,9,10$ with $457,437,17,13$, and 7 segments. Bottom: CLMC diffusion, $k=0,1,3,4,5$ with 176, 157, 129, 17, and 7 segments.

iii) To illustrate the robustness of the dynamics of contours in scale-space a comparison among hierarchies is presented via the saliency maps shown in Fig. 8. These maps are obtained using (a) the dynamics of contours at the localization scale $\left(D C^{0}=\right.$ superficial image structure), (b) the scale-space lifetime ( $S L T=$ deep image structure), and (c) the dynamics of contours in scale-space (DCS).

For all three saliency maps, the results obtained using the $C L M C$ diffusion are, as expected, more meaningful. Furthermore, the integration of the superficial with the deep image structure using the DCS principle allows refinement and optimization of the hierarchy.

Note that, the saliency map obtained using only the superficial image structure [Fig. 8(a)] resembles the segmentation method presented in [31] while the one obtained using the scalespace lifetime [Fig. 8(b)] in the case of Gaussian diffusion resembles the segmentation method presented in [7]. 


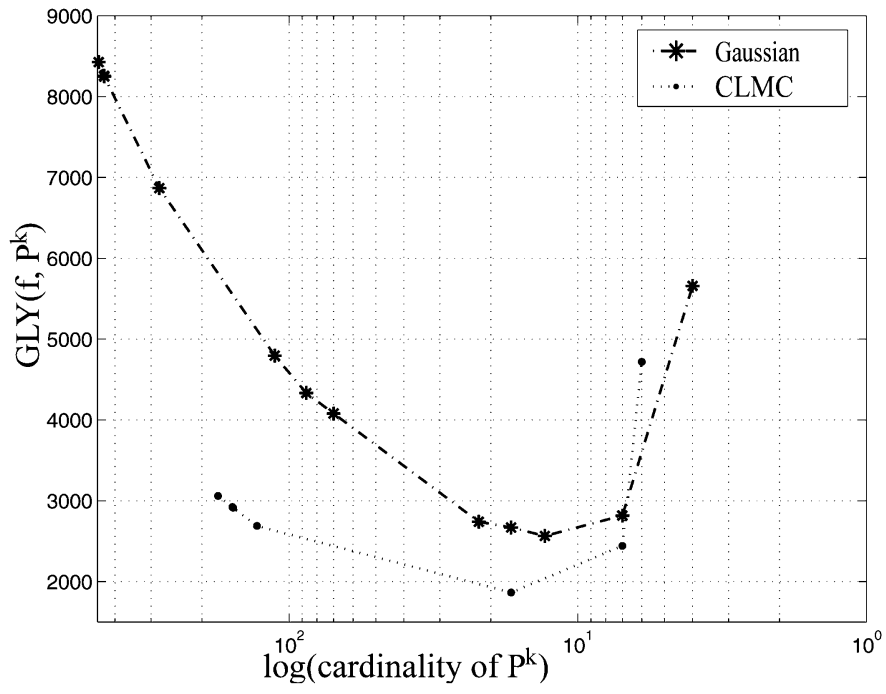

Fig. 10. Segmentation evaluation measure of the hierarchical levels.

iv) The hierarchical level retrieval module identifies a set of segmented images with different levels of abstraction. Fig. 9 illustrates the obtained hierarchical levels using either Gaussian or $C L M C$ diffusion. In these figures, the set of the produced hierarchical levels is shown. As it can be seen, in the case of using the $C L M C$ diffusion, less and more relevant hierarchical levels are obtained.

The proposed segmentation scheme enables an automatic selection of an optimal hierarchical level (optimal segmentation) using a segmentation quality measure inspired by the evaluation measure proposed by Liu and Yang in [11]. It relies on i) intra-segment uniformity, ii) inter-segment contrast, and iii) smoothness of the boundaries between segments. It is given by

$$
\mathcal{G} \mathcal{L} \mathcal{Y}\left(\mathbf{f}, P^{k}\right)=\sqrt{n_{k}} \sum_{S_{i}^{k} \in P^{k}} \sum_{\mathbf{x}_{j} \in S_{i}^{k}} d_{E}^{2}\left(\mathbf{f}\left(\mathbf{x}_{j}\right), P^{k}\left(\mathbf{x}_{j}\right)\right)
$$

where $\mathbf{f}$ is the color image, $P^{k}$ is the partitioning at the hierarchical level $k, n_{k}$ denotes the number of segments at level $k$, and $d_{E}(.,$.$) is the Euclidean distance. The term \sqrt{n_{k}}$ is a global measure which penalizes segmentations with too many segments. The term $\sum d_{E}^{2}$ is a local measure which penalizes segments with a large color error. Eq. (15) expresses the trade-off between the suppression of heterogeneity and the preservation of detail. The smaller the value of $\mathcal{G} \mathcal{L Y}\left(\mathbf{f}, P_{k}\right)$, the better the segmentation result is, as it is shown in Fig. 10.

In Fig. 11, the optimal level in the hierarchical tree according to the $\mathcal{G} \mathcal{L Y}$ evaluation criterion is shown for a number of test images. For these images, we provide Table I that illustrates the number of hierarchical levels and the number of regions in the optimal hierarchical level in the case of Gaussian and CLMC diffusion.

Our experiments have shown that the optimal hierarchical level using the CLMC diffusion often contains more segments compared to the number of segments obtained using the Gaussian diffusion. However, the segmentation scheme based on the $C L M C$ diffusion leads to a more accurate segmentation with high detail and improved visual quality.
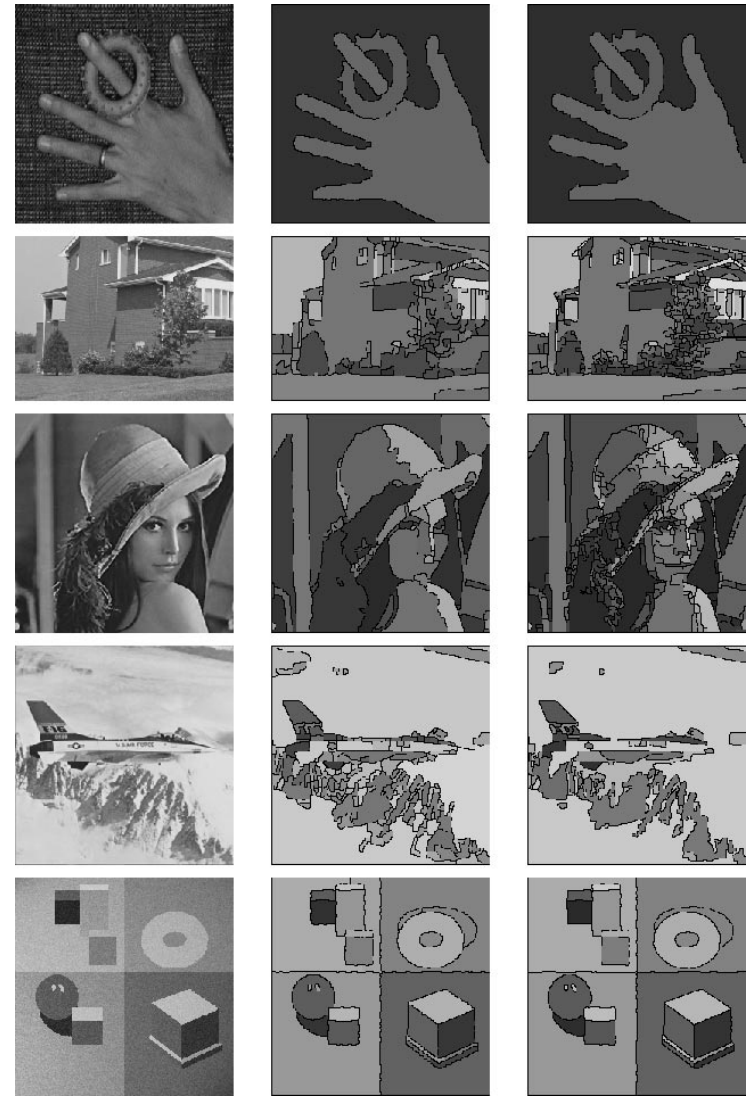

Fig. 11. Optimal hierarchical level selection. Left column: original images. Middle column: Gaussian diffusion. Right column: CLMC diffusion.

TABLE I

SEGMENTATION RESULTS

\begin{tabular}{l|r|r|r|r|r|r}
\hline & \multicolumn{2}{|c|}{$\begin{array}{c}\text { Number of } \\
\text { hierarchical } \\
\text { levels }\end{array}$} & \multicolumn{2}{c|}{$\begin{array}{c}\text { Optimal } \\
\text { hierarchical } \\
\text { level }\end{array}$} & \multicolumn{2}{c}{$\begin{array}{c}\text { Number } \\
\text { of } \\
\text { segments }\end{array}$} \\
\hline Image & Gauss. & CLMC & Gauss. & CLMC & Gauss. & CLMC \\
\hline girl & 12 & 7 & 9 & 4 & 13 & 17 \\
hand & 8 & 8 & 6 & 7 & 6 & 6 \\
house & 11 & 6 & 6 & 3 & 126 & 349 \\
lena & 8 & 8 & 5 & 3 & 77 & 301 \\
plane & 9 & 8 & 7 & 6 & 192 & 91 \\
toys & 8 & 10 & 5 & 5 & 30 & 26 \\
\hline
\end{tabular}

\section{CONCLUSIONS}

Watershed-driven segmentation is a powerful tool in morphological segmentation once we can deal with the oversegmentation. The proposed multiscale method to deal with this problem is very versatile. It employs a key characteristic of the color gradient watershed and integrates the deep image structure with the superficial image structure. In this paper, we demonstrated that the use of an anisotropic scale-space is beneficial when dealing with small but salient features and when boundary location is critical.

In future work, we intend to improve the numerical scheme by using a version of the $A O S$ numerical scheme that relaxes the quality restriction on the time-step [52]. The scale-space stack generation shall be automated by adding a module that detects convergence and by using a scale selection method for 
the localization scale [53]. Furthermore, a theoretical study of the evolution of the top-points of a color gradient in CLMC scale-space shall be carried out and a better estimation of the gradient for the diffusivity shall be investigated [50]. These can lead to improvements of the scale sampling (scale selection) and the parent-child linking process. Additionally, the use of other color spaces and scale-spaces, methods for the integration of the scale-information and other salient measures will be investigated. Furthermore, as the proposed multiscale method contains several hierarchies among the gradient watershed, it is useful to investigate methods to combine multiple hierarchies [24] to improve the extraction of the relevant hierarchical levels.

\section{REFERENCES}

[1] N. R. Pal and S. K. Pal, "A review on image segmentation techniques," Pattern Recognition, vol. 26, no. 9, pp. 1277-1294, 1993.

[2] D. Mumford and J. Shah, "Optimal approximation by piecewise smooth functions and associated variational problems," Comm. Pure Appl. Math., vol. 42, no. 4, pp. 577-685, 1989.

[3] J. M. Morel and S. Solimini, "Variational methods in image segmentation," in Progress in Nonlinear Differential Equations and Their Applications. Cambridge, MA: Birkhaüser, 1995.

[4] J. Weickert, "Fast segmentation methods based on partial diffierential equations and the watershed transformation," in Mustererkennung, P. Levi et al., Eds. Berlin, Germany: Springer, 1998, pp. 93-100.

[5] J. M. Gauch and S. M. Pizer, "Multiresolution analysis of ridges and valleys in grey-scale images," IEEE Trans. Pattern Anal. Machine Intell., vol. 15, no. 6, pp. 635-646, 1993.

[6] I. Pratikakis, H. Sahli, and J. Cornelis, "Hierarchical segmentaion using dynamics of multiscale gradient watersheds," in 11th Scandinavian Conf. on Image Analysis (SCIA 99), 1999, pp. 577-584.

[7] O. F. Olsen and M. Nielsen, "Multi-scale gradient magnitude watershed segmentation," in Int. Conf. on Image Analysis and Processing (ICIAP '97), 1997, pp. 6-13.

[8] P. T. Jackway, "Gradient watershed in morphological scale-space," IEEE Trans. Image Processing, vol. 5, pp. 913-921, June 1996.

[9] J. Crespo and R. W. Schafer, "The flat zone approach and color images," in Mathematical Morphology and Its Applications to Image Processing, J. Serra et al., Eds. Norwell, MA: Kluwer, 1994, pp. 85-92.

[10] C. H. Demarty and S. Beucher, "Color segmentation using an HLS transformation," in Mathematical Morphology and Its Applications to Iimage Processing, H. Heijmans et al., Eds. Norwell, MA: Kluwer, 1998, pp. 231-238.

[11] J. Liu and Y.-H. Yang, "Multiresolution color image segmentation," IEEE Trans. Pattern Anal. Machine Intell., vol. 16, no. 7, pp. 689-700, 1994.

[12] F. Meyer, "Color image segmentation," in Proc. 4th IEEE Conf. on Image Processing and Apllications, vol. 354, 1992, pp. 303-306.

[13] K. Saarinen, "Watersheds in color image segmentation," in Proc. IEEE Workshop on Nonlinear Signal and Image Processing, 1995, pp. 14-17.

[14] L. Lucchese and S. K. Mitra, "Color segmentation based on separate anisotropic diffusion of chromatic and achromatic channels," Proc. IEEE, vol. 148, pp. 141-150, Mar. 2001.

[15] P. De Smet, R. Pires, D. De Vleeschauwer, and I. Bruyland, "Activity driven nonlinear diffusion for color image watershed segmentation," SPIE Journal of Electronic Imaging, Special Section on Nonlinear and Model-Based Image Analysis, vol. 8, no. 3, pp. 270-278, 1999.

[16] I. Vanhamel, "Automatic watershed segmentation of color images," Tech. Rep. TR0058, Vrije Universiteit Brussel, Nov. 1999.

[17] I. Vanhamel, I. Pratikakis, and H. Sahli, "Automatic watershed segmentation of color images," in Mathematical morphology and its applications to image processing, J. Goutsias et al., Eds: Kluwer Academic Publishers, 2000, pp. 207-214.

[18] I. Vanhamel, I. Pratikakis, and H. Sahli, "Hierarchical segmentation using dynamics of multiscale color gradient watersheds," in Scale-Space and Morphology in Computer Vision, M. Kerckhove, Ed. New York: Springer, 2001, pp. 371-379.

[19] S. Makrogiannis, I. Vanhamel, S. Fotopoulos, and H. Sahli, "Scale space segmentation of color images using watersheds and fuzzy region merging," in Proc. Int. Conf. on Image Processing, vol. I, Thessaloniki, Oct. 2001, pp. 734-737.
[20] A. Brook, R. Kimmel, and N. Sochen, "Geometry motivated variational segmentation for color images," in Scale-Space and Morphology in Computer Vision, M. Kerckhove, Ed. New York: Springer, 2001, pp. 362-370.

[21] K. L. Vincken, A. S. E. Koster, and M. A. Viergever, "Probabilistic multiscale image degmentation," IEEE Trans. Pattern Anal. Machine Intell., vol. 19, no. 2, pp. 109-120, 1997.

[22] J. M. Gauch, "Image segmentation and analysis via multiscale gradient watershed hierarchies," IEEE Trans. Image Processing, vol. 8, pp. 69-79, Jan. 1999.

[23] M. Mirmehdi and M. Petrou, "Segmentation of color textures," IEEE Trans. Pattern Anal. Machine Intell., vol. 22, no. 2, pp. 142-159, 2000.

[24] F. Meyer, "Hierarchies of partitions and morphological segmentation," in Scale-Space and Morphology in Computer Vision, M. Kerckhove, Ed. New York: Springer, 2001, pp. 161-182.

[25] P. Salembier and J. Serra, "Flat zone filtering, connected operators, and filters by reconstruction," IEEE Trans. Image Processing, vol. 4, pp. 1153-1160, Aug. 1995.

[26] F. Meyer, A. Oliveras, P. Salembier, and C. Vachier, "Morphological tools for segmentations: Connected filters and watershed," Ann. Telecommun., pp. 367-380, 1997.

[27] A. S. Wright and S. T. Acton, "Watershed pyramids for edge detection," in Proc. Int. Conf. on Image Processing, vol. II, Washington, DC, Oct. 1997.

[28] J. Bosworth, T. Koshimizu, and S. T. Acton, "Automated segmentation of surface soil moisture from landsat tm data," in Proc. IEEE Southwest Symp. Image Analysis and Interpretation, Tuscon, AZ, 1998, pp. 70-74.

[29] I. Pratikakis, "Watershed-driven image segmentation," Ph.D. disertation, Vrije Univ. Brussels, Brussels, Belgium, 1998.

[30] R. Whitaker and G. Gerig, "Vector-valued diffusion," in Geometry-Driven Diffusion in Computer Vision, B. M. ter Haar Romeny, Ed. Norwell, MA: Kluwer, 1994, pp. 93-134.

[31] L. Najman and M. Schmitt, "Geodesic saliency of watershed contours and hierarchical segmentation," IEEE Trans. Pattern Anal. Machine Intell., vol. 18, pp. 1163-1173, Dec. 1996.

[32] P. Perona and J. Malik, "Scale-space and edge detection using anisotropic diffusion," IEEE Trans. Pattern Anal. Machine Intell., vol. 12, no. 7, pp. 629-639, 1990.

[33] F. Catté, P.-L. Lions, J.-M. Morel, and T. Coll, "Image selective smoothing and edge detection by nonlinear diffusion," SIAM J. Numer. Anal., vol. 29, no. 1, pp. 182-193, 1992.

[34] J. Weickert, Anisotropic Diffusion in Image Processing. Stuttgart, Germany: Teubner-Verlag, 1998.

[35] J. Weickert, B. M. ter Haar Romeny, and M. A. Viergever, "Efficient and reliable schemes for nonlinear diffusion filtering," IEEE Trans. Image Processing, vol. 7, pp. 398-410, Mar. 1998.

[36] S. Beucher, "Segmentation d'images et morphologie mathématique," Ph.D. dissertation, Ecole nationale supérieure des mines de Paris, Fontainebleau, 1990.

[37] M. Grimaud, "A new measure of contrast: The dynamics," in Proc. SPIE Image Algebra and Morphological Image Processing III San Diego, CA, 1992, vol. 1769, pp. 292-305.

[38] C. Vachier and F. Meyer, "Extinction value: A new measurement of persistence," in Proc. IEEE Workshop on Nonlinear Signal and Image Processing, June 1995, pp. 254-257.

[39] S. Beucher, "Watershed, hierarchical segmentation and waterfall algorithm," in Mathematical Morphology and Its Applications to Image Processing, J. Serra et al., Eds. Norwell, MA: Kluwer, 1994, pp. 69-76.

[40] L. Vincent and P. Soille, "Watersheds in digital spaces: An efficient algorithm based on immersion simulations," IEEE Trans. Pattern Anal. Machine Intell., vol. 13, pp. 583-598, 1991.

[41] S. Beucher and C. Lantuejoul, "Use of watersheds in contour detection," in Proc. Int. Workshop on Image Processing, Real-Time Edge and Motion Detection/Estimation, Rennes, France, 1979.

[42] L. Shafarenko, M. Petrou, and J. Kittler, "Automatic watershed segmentation of randomly textured color images," IEEE Trans. Image Processing, vol. 6, pp. 1530-1544, Nov. 1997.

[43] J. N. Hwang, S. R. Lay, and A. Lippman, "Nonparametric multivariate density estimation: A comparative study," IEEE Trans. Signal Processing, vol. 42, pp. 2795-2810, Oct. 1994.

[44] S. Di Zenzo, "A note on the gradient of a multi-image," Comput. Vis., Graph., Image Process., vol. 33, pp. 116-125, 1986.

[45] T. Gevers and A. W. M. Smeulders, "Pictoseek: Combining color and shape invariant features for image retrieval," IEEE Trans. Image Processing, vol. 9, pp. 102-119, Jan. 2000.

[46] J. J. Koenderink, "The structure of images," Biol. Cybern., vol. 50, pp. 363-370, 1984. 
[47] T. Lindeberg, Scale-Space Theory in Computer Vision. Dordrecht, The Netherlands: Kluwer, 1994

[48] J. Sporring and O. F. Olsen, "Segmenting by compression using linear scale-space and watersheds," in Proc. Int. Conf. on Scale Space Theories in Computer Vision, 1999, pp. 513-518.

[49] A. Chambolle, "Partial differential equations and image processing," in Proc. Int. Conf. on Computer Vision, vol. 1, Austin, TX, 1994, pp. 16-20.

[50] G. Sapiro and D. L. Ringach, "Anisotropic diffusion of multivalued images with applications to color filtering," IEEE Trans. Image Processing, vol. 5, pp. 1583-1586, Nov. 1996.

[51] G. Sapiro, Geometric Partial Differential Equations and Image Processing. Cambridge, U.K.: Cambridge Univ. Press, 2001.

[52] D. Barash, M. Israeli, and R. Kimmel, "An accurate operator splitting scheme for nonlinear diffusion filtering," Scale-Space and Morphology in Computer Vision, pp. 281-289, 2001.

[53] Z. Lin and Q. Shi, "An anisotropic diffusion pde for noise reduction and thin edge preservation," in Proc. Int. Conf. on Image Analysis and Processing, Venice, Italy, 1999, pp. 102-107.

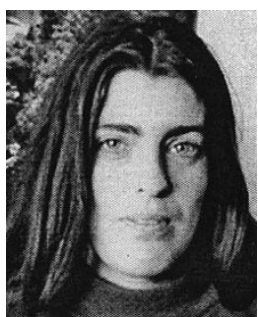

Iris Vanhamel was born in Genk, Belgium, in 1973. She received the M.S. degree in in electrotechnical engineering and information processing at the Vrije Universiteit Brussels (VUB), Brussels, Belgium, in 1998. She is currently pursuing the Ph.D. degree at the Department of Electronics and Information Processing (ETRO), VUB.

Her research interest include image segmentation, mathematical morphology, scale-space theory, and multispectral image processing.

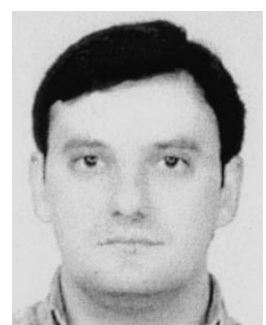

Ioannis Pratikakis (M'00) received the degree in electrical engineering from the Demokritus University of Thrace, Xanthi, Greece, as well as a Ph.D. degree in Applied Sciences from Vrije Universiteit Brussel, Brussels, Belgium, in 1992 and 1998, respectively.

From March 1999 to March 2000, he was at IRISA/ViSTA group, Rennes, France, as a Postdoctoral Fellow. He is currently working as a Research Associate at the Institute of Informatics and Telecommunications of the National Center for Scientific Research "Demokritos," Athens, Greece. His research interests include mathematical morphology, scale-space theory, image registration, color image processing and 3-D reconstruction with applications in the field of medicine and robotics.

Dr. Pratikakis is a member of the Hellenic Technical Chamber.

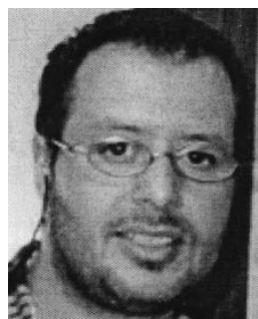

Hichem Sahli (A'00) was born in Tunis, Tunisia, in 1960.

$\mathrm{He}$ is currently Professor of image analysis and computer vision with the Department of Electronics and Information Processing, Vrije Universiteit Brussel, Brussels, Belgium. He coordinates the research team in computer vision. His research interests include image analysis and interpretation, computer vision, mathematical morphology, scale-space theory, image registration, image and 3-D reconstruction. 